\title{
Investigation of the presence of G354A (Cys87Tyr) mutation in osteoprotegerin gene in women with osteoporosis in Chaharmahal and Bakhtiari province
}

\author{
Seyedeh Zahra Mousavi ${ }^{1 \mathbb{D}}$, Morteza Dehghan $^{2}$, Razieh Pourahmad $^{1 *(\mathbb{D})}$ \\ 'Department of Genetics, Faculty of Science, Shahrekord University, Shahrekord, Iran \\ ${ }^{2}$ Department of Orthopedics, Shahrekord University of Medical Sciences, Shahrekord, Iran
}

*Corresponding Author: Razieh Pourahmad, Tel/Fax: 03832324419, E-mail: razieh_jaktaji@yahoo.com

\begin{abstract}
Background and aims: Osteoprotegerin (OPG) is a competitive inhibitor of the differentiation and activity of osteoclasts, which inhibits the final stages of osteoclast formation and induces its apoptosis. In addition, OPG is considered as one of the most important candidate genes in the pathogenesis of bone diseases such as osteoporosis and idiopathic hyperphosphatasia. The G354A (Cys87Tyr) mutation in the OPG gene leads to idiopathic hyperphosphatasia. This mutation is probably related to osteoporosis. The purpose of this study was to investigate the presence of G354A (Cys87Tyr) in women with osteoporosis in Chaharmahal and Bakhtiari province.

Methods: In this descriptive-analytical study, the bone mineral density (BMD) of the femoral neck and lumbar spine of women referring to Shahrekord bone densitometry centers was measured by the X-ray absorptiometry technique in 2013-2014. Based on T-scores, people with osteoporosis were identified and 70 patients were enrolled in the study after receiving their consent. Finally, DNA was extracted from blood samples, amplified by polymerase chain reaction (PCR) technique, and sequenced by DNA sequencing method.

Results: After DNA extraction from the blood, the quality and quantity were determined by gel electrophoresis and spectrophotometry, respectively. Then, the gene was amplified by the PCR method and the product was detected by gel electrophoresis, followed by sequencing the samples to investigate the presence of the mutation. Eventually, genotypes associated with Cys87Tyr mutation were not observed in the studied population.

Conclusion: In the present study, the G354A (Cys87Tyr) mutation associated with idiopathic hyperphosphatasia was not found in women with osteoporosis.

Keywords: G354A mutation, OPG gene, Osteoporosis
\end{abstract}

Received: 20 May 2019, Accepted: 2 July 2019, ePublished: 31 December 2019

\section{Introduction}

Osteoporosis is regarded as a skeletal disease that is associated with decreased bone mass and increased risk of bone fragility (1). Previous evidence indicates that on average, over $50 \%$ of women over the age of 50 are at the risk of osteoporotic fractures (2).

The key mechanism that regulates the rate of bone formation and resorption (regeneration) is related to the RANKL/RANK/OPG pathway. In fact, RANKL, its receptor (RANK), and its decoy receptor osteoprotegerin (OPG) play a key role in regulating bone regeneration.

This pathway is based on the increased differentiation of osteoclasts through the binding of the RANKL ligand to its membrane receptor (RANK) on mononuclear osteoclast precursors. This increased differentiation of osteoclasts by RANKL is inhibited by the OPG that is produced by osteoblasts (3).

In the bone microenvironment, the ratio of RANKL to OPG indicates the activation rate of the osteoclasts. In addition, the normal activation of osteoclasts is due to the physiological degradation of bone in bone regeneration while the excessive activity of osteoclasts leads to various bone diseases including osteoporosis (4).

According to some studies, many mutations and singlenucleotide polymorphisms in the human OPG gene are associated with osteoporosis or osteoporosis phenotypes, including osteoporotic fractures and decreased bone mineral density (BMD), as well as other metabolic and bone regeneration-related diseases such as idiopathic hyperphosphatasia $(5,6)$. Idiopathic hyperphosphatasia is a rare autosomal recessive bone disease and is observed in young male and female children (7). There is a considerable phenotypic variation from severe to mild in the affected people.

Generally, babies are normal at birth, but they gradually exhibit bone radiological changes, bone fractures, bone

(C) 2019 The Author(s); Published by Shahrekord University of Medical Sciences. This is an open-access article distributed under the terms of the Creative Commons Attribution License (http://creativecommons.org/licenses/by/4.0), which permits unrestricted use, distribution, and reproduction in any medium, provided the original work is properly cited. 
pain, and skull enlargement, as well as the increased risk of pathologic fractures and non-bone changes (8).

Biochemical analyses represent that there is a significant increase in the serum alkaline phosphatase activity, which results in an increase in the rate of bone regeneration $(6,8)$. Previous research demonstrates that the disease is mainly due to the mutation in the OPG coding gene. In this disease, the loss of normal OPG function leads to increased bone regeneration (6). The mutation G354A (Cys87Tyr) located in exon 2 of the OPG gene leads to idiopathic hyperphosphatasia possibly due to the degradation of the sulfide bonds in the second cysteine-rich domain II (the second most effective domain in interacting with the ligand) OPG and the reduction of cysteine amino acids (6).

Moreover, the loss of the disulfide bond apparently leads to the loss of the natural structure of OPG and the disruption of OPG binding to its ligand (RANKL). The resulting changes in protein structure may also affect protein stability $(6,9)$.

This homozygous missense mutation has been investigated in different countries and was observed in a six-year-old boy with idiopathic hyperphosphatasia in Argentina (6). However, another recessive missense mutation (C65R) in cysteine residue of exon 2 was reported in a girl in Turkey (6). Although the BMD of their parents, who were the carrier of these mutations, were not assessed, the heterozygous form of Cys87Tyr mutation may be associated with BMD.

Therefore, the current study sought to evaluate the presence of G354A mutation (Cys87Tyr) for the first time in women with osteoporosis in Chaharmahal and Bakhtiari province.

\section{Materials and Methods}

In this descriptive-analytical study, the BMD of the lumbar spine and the femoral neck was measured in women referring to Shahrekord bone densitometry centers by the X-ray absorptiometry method and Hologic QDR device (Germany) in 2013-2014.

Patients with a history of corticosteroid use, ovariectomy or early ovarian failure, thyroid disease, impaired calcium absorption, as well as digestive and kidney diseases were excluded from the study.

People with osteoporosis were identified based on T-score, 70 of whom were enrolled in the study after completing the consent form for participation in the study.

For genetic assessments, a 5-mL complete blood sample of each participant was collected by the simple sampling method and then poured into a tube containing the anticoagulant ethylenediaminetetraacetic acid. The genomic DNA was extracted from the blood samples using the protocol of the CinnaGen kit. The concentration of the extracted DNA and its quality were determined using a spectrophotometer and agarose gel electrophoresis, respectively.
The desired range of the OPG gene was amplified using the primers designed by Gene Runner software. Further, the sequences of forward and reverse primers were 5'-GTACAGCAAAGTGGAAGA-3' and 5'-CTCCTAAACTGTCACAACTA-3', respectively. The polymerase chain reaction (PCR) solution included $2.5 \mu \mathrm{L}$ buffer 10X, $1.5 \mu \mathrm{L} 50 \mathrm{mM} \mathrm{MgCl} 2,1 \mu \mathrm{L} 40 \mathrm{mM}$ dNTP Mix, $0.7 \mu \mathrm{L}$ forward primer $10 \mathrm{pmol} / \mathrm{mL}, 0.2 \mu \mathrm{L}$ Taq polymerase $5 \mathrm{U} / \mu \mathrm{L}, 3.2 \mu \mathrm{L}$ template $\mathrm{DNA}$, and $\mathrm{ddH}_{2} \mathrm{O}$ to a final reaction volume of $25 \mu \mathrm{L}$. PCR reactions were performed using the thermocycler model (ASTEC, PC818 Japan) with the thermal conditions as follows: $95^{\circ} \mathrm{C}$ for 5 minutes, followed by 36 cycles of $94^{\circ} \mathrm{C}, 45^{\circ} \mathrm{C}$, and $72^{\circ} \mathrm{C}$ for 30 , 30 , and 30 seconds, respectively, with a final extension at $72^{\circ} \mathrm{C}$ for 10 minutes.

The length of the amplified fragment was $308 \mathrm{bp}$ and then the fragment was observed by gel electrophoresis. Finally, the samples were sequenced by Genfanavaran Company to investigate the presence of a mutation.

\section{Results}

People with a T-score equal to -2.5 or higher are at the risk of osteoporosis. Based on this criterion, 70 patients with osteoporosis were identified and then enrolled in the study after receiving their consent. After PCR amplification, the product size was examined on the agarose gel. The image of the PCR products is shown in Figure 1.

The samples were sequenced to determine the presence of the G354A (Cys87Tyr) mutation and the sequencing results for all 70 patients showed normal allele while demonstrating no mutation (Figure 2).

\section{Discussion}

Osteoporosis is a multifactorial (multigenic and multienvironmental) disease in postmenopausal women (1). It is characterized by an increase in fracture risk and a reduction in bone density (2). Genetic factors play important roles in the pathogenesis of osteoporosis and reduction of BMD. The examples of these genetic factors are vitamin D receptor, collagen 1a1, and OPG genes (1012). It was suggested that the sequence variation in genes other than OPG have a substantial impact on BMD, bone regeneration rate, and osteoporosis (13). Furthermore, the OPG gene is associated with osteoporosis or osteoporosis phenotypes such as osteoporotic fractures and decreased BMD, along with other metabolic and bone regenerationrelated diseases such as idiopathic hyperphosphatasia $(4,6,13)$. This gene contains various polymorphic sites. The association of some of these polymorphic sites with BMD and osteoporosis was investigated in different races and geographical locations $(13,14)$. Some of these studies show contradictory results in this regard. There are seven polymorphic sites (i.e., Ilu16Thr, Thr20Ilu, Cys87Tyr, Val104Met, Phe117Leu, Cys87Tyr, Cys65Arg) in exon 2 of the OPG gene $(6,10,15-18)$. Four of them 


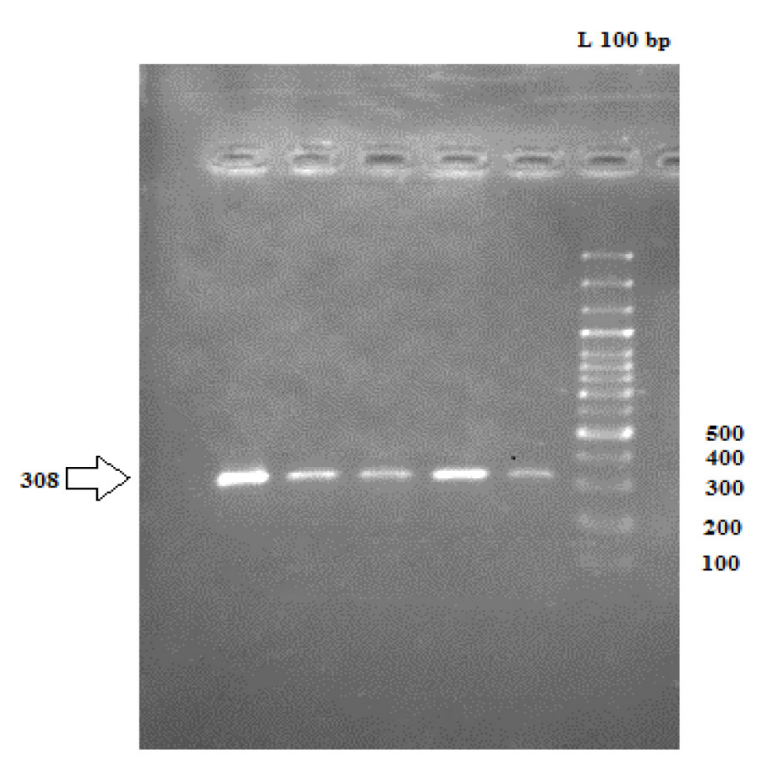

Figure 1. The Agarose Gel of PCR Products.

were mainly studied in Chinese postmenopausal women and the findings confirmed their associations with BMD $(10,15-17)$. Three other sites were indicated to be related to idiopathic hyperphosphatasia phenotypes (severe to mild phenotypes), including deformities and fractures in two girls and one boy in Argentina and Turkey (6). One of these polymorphic sites (Phe117Leu), which was observed in an idiopathic hyperphosphatasia girl in Argentina, was also studied in Chinese women and claimed to be associated with BMD (18). The Cys87Tyr site, which was related to severe phenotype in an idiopathic hyperphosphatasia boy with homozygote form (Tyr/Tyr), may also be a good candidate for investigating the possible association with BMD. Also, sex may have an effect in association studies as with the $\mathrm{T}^{950} \mathrm{C}$ polymorphism in Swedish women and men populations $(19,20)$. Considering the above-mentioned explanations, it was decided to study the presence of G354A mutation (Cys87Tyr) in women with osteoporosis in Chaharmahal and Bakhtiari Province.

Due to the importance of OPG in bone metabolism, the heterozygote form of this site (Cys/Tyr) could be found in osteoporotic women. However, the findings of this study showed only one homozygote phenotype (Cys/Cys), while the carrier of this mutation was not found in this group. This is consistent with the previous study in which it is considered a rare change in the exon 2 of OPG. Although it was reported that the G354A (Cys87Tyr) change is a rare mutation, the presence of this mutation was investigated in the healthy group. Moreover, no carrier of this mutation was reported in this group in the current study (data not available).

However, given that a wide variety of genetic and environmental factors play a role in the development
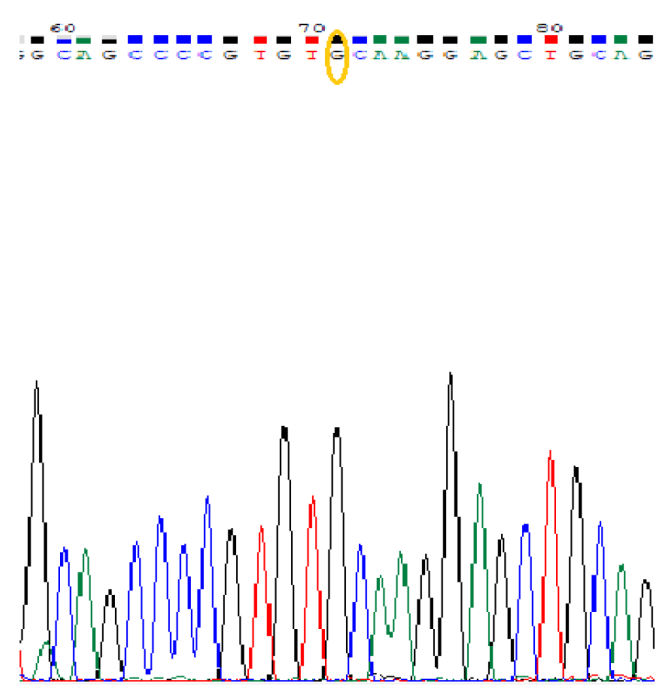

Figure 2. DNA sequencing result and position of G354A mutation in the osteoprotegerin gene.

Note. The same results were obtained for all samples which were homozygote (GG).

of bone diseases such as osteoporosis and idiopathic hyperphosphatasia and limited research is available on this mutation, future studies are recommended to investigate the presence of this mutation in people (men and women) with osteoporosis and other bone diseases. Moreover, the mutation in other genes like RANK, CSF1, and TM7SF4 related to idiopathic hyperphosphatasia $(7,8)$ can also be evaluated in osteoporotic males and females.

\section{Conclusion}

In our study, the G354A (Cys87Tyr) mutation associated with idiopathic hyperphosphatasia was not observed in women with osteoporosis in Chaharmahal and Bakhtiari Province. Accordingly, further studies on this mutation in OPG and other genes such as RANK, CSF1, and TM7SF4 are recommended to be conducted on people (men and women) with bone diseases.

Conflict of interests

None.

Ethical considerations

The study protocol was approved at the Research Ethics Committee of Shahrekord University of Medical Sciences (IR.SKUMS. REC.1395.74).

\section{Acknowledgements}

Hereby, we would like to thank the Research Deputy of Shahrekord University, as well as the Research and Technology Deputy of Shahrekord University of Medical Sciences (grant No: 2093).

\section{References}

1. Tu KN, Lie JD, Wan CKV, Cameron M, Austel AG, Nguyen JK, et al. Osteoporosis: a review of treatment options. P T. 2018;43(2):92-104. 
2. Eastell R, O'Neill TW, Hofbauer LC, Langdahl B, Reid IR, Gold DT, et al. Postmenopausal osteoporosis. Nat Rev Dis Primers. 2016;2:16069. doi: 10.1038/nrdp.2016.69.

3. Nozawa S, Inubushi T, Irie F, Takigami I, Matsumoto K, Shimizu $\mathrm{K}$, et al. Osteoblastic heparan sulfate regulates osteoprotegerin function and bone mass. JCI Insight. 2018;3(3). doi: 10.1172/ jci.insight.89624.

4. Luan X, Lu Q, Jiang Y, Zhang S, Wang Q, Yuan H, et al. Crystal structure of human RANKL complexed with its decoy receptor osteoprotegerin. J Immunol. 2012;189(1):245-52. doi: 10.4049/jimmunol.1103387.

5. Kohli SS, Kohli VS. Role of RANKL-RANK/osteoprotegerin molecular complex in bone remodeling and its immunopathologic implications. Indian J Endocrinol Metab. 2011;15(3):175-81. doi: 10.4103/2230-8210.83401.

6. Chong B, Hegde $M$, Fawkner $M$, Simonet $S$, Cassinelli $H$, Coker $\mathrm{M}$, et al. Idiopathic hyperphosphatasia and TNFRSF11B mutations: relationships between phenotype and genotype. J Bone Miner Res. 2003;18(12):2095-104. doi: 10.1359/ jbmr.2003.18.12.2095.

7. Donáth J, Speer G, Kósa JP, Árvai K, Balla B, Juhász P, et al. Polymorphisms of CSF1 and TM7SF4 genes in a case of mild juvenile Paget's disease found using next-generation sequencing. Croat Med J. 2015;56(2):145-51. doi: 10.3325/ cmj.2015.56.145.

8. Kumar SRR, Bagalad BS, Manohar CB, Kuberappa PH. Intermediate Type of Juvenile Paget's Disease: A Rare Case in Indian Population. Contemp Clin Dent. 2017;8(1):175-8. doi: 10.4103/ccd.ccd_1097_16.

9. Papadopouli AE, Klonaris CN, Theocharis SE. Role of OPG/ RANKL/RANK axis on the vasculature. Histol Histopathol. 2008;23(4):497-506. doi: 10.14670/hh-23.497.

10. Zhu J, Luo Z, Cao Y, Yu M, Peng J, Huang D. The influence of g.19124G >a genetic polymorphism in the OPG gene on bone mineral density in Chinese women. Genet Test Mol Biomarkers. 2013;17(9):696-9. doi: 10.1089/gtmb.2013.0183.

11. Dehghan M, Pourahmad-Jaktaji R. Sp1 binding site polymorphism of a collagen gene (rs 1800012) in women aged 45 and over and its association with bone density. Turk J Med
Sci. 2015;45(3):644-50. doi: 10.3906/sag-1405-80.

12. Dehghan $M$, Pourahmad-Jaktaji R. The effect of some polymorphisms in vitamin $\mathrm{D}$ receptor gene in menopausal women with osteoporosis. J Clin Diagn Res. 2016;10(6):RC0610. doi: 10.7860/jcdr/2016/17147.8006.

13. Langdahl BL, Carstens $M$, Stenkjaer L, Eriksen EF. Polymorphisms in the osteoprotegerin gene are associated with osteoporotic fractures. J Bone Miner Res. 2002;17(7):1245-55. doi: 10.1359/jbmr.2002.17.7.1245.

14. Arko B, Prezelj J, Komel R, Kocijancic A, Hudler P, Marc J. Sequence variations in the osteoprotegerin gene promoter in patients with postmenopausal osteoporosis. J Clin Endocrinol Metab. 2002;87(9):4080-4. doi: 10.1210/jc.2002-020124.

15. Yu F, Huang X, Miao J, Guo L, Tao D. Association between osteoprotegerin genetic variants and osteoporosis in Chinese postmenopausal women. Endocr J. 2013;60(12):1303-7. doi: 10.1507/endocrj.ej13-0308.

16. Wang F, Cao Y, Li F, Shan J, Wen T. Association analysis between g.18873C $>T$ and g.27522G $>A$ genetic polymorphisms of OPG and bone mineral density in Chinese postmenopausal women. Biomed Res Int. 2014;2014:320828. doi: 10.1155/2014/320828.

17. Zhang YD, Zhang Z, Zhou NF, Jia WT, Cheng XG, Wei XJ. Association of the g.19074G>A genetic variant in the osteoprotegerin gene with bone mineral density in Chinese postmenopausal women. Genet Mol Res. 2014;13(3):6646-52. doi: 10.4238/2014.August.28.9.

18. Liu S, Yi Z, Ling M, Shi J. Association between g.19163A>G and g.23298T>C genetic variants of the osteoprotegerin gene and bone mineral density in Chinese women. Hormones (Athens). 2013;12(4):578-83. doi: 10.14310/horm.2002.1446.

19. Brandstrom H, Stiger F, Michaelsson K, Gillberg P, Ljunghall S, Ljunggren $\mathrm{O}$, et al. Polymorphism in the promoter region of the human gene for osteoprotegerin: correlation with bone mineral density. J Bone Miner Res. 1999;14:S334.

20. Brandstrom H, Gerdhem P, Stiger F, Obrant K, Ljunggren $\mathrm{O}$, Kindmark A. Polymorphisms in the genes for vitamin D receptor and osteoprotegerin, relation to bone mineral density in Swedish women aged 75. J Bone Miner Res. 2000; 15: S262. 